\title{
Energetics and geometry of excitations in random systems
}

\author{
A. Alan Middleton \\ Department of Physics, Syracuse University, Syracuse, New York 13244
}

(July 24, 2000)

\begin{abstract}
Methods for studying droplets in models with quenched disorder are critically examined. Low energy excitations in two dimensional models are investigated by finding minimal energy interior excitations and by computing the effect of bulk perturbations. The numerical data support the assumptions of compact droplets and a single exponent for droplet energy scaling. Analytic calculations show how strong corrections to power laws can result when samples and droplets are averaged over. Such corrections can explain apparent discrepancies in several previous numerical results for spin glasses.

75.10.Nr, 74.60.Ge, 02.70.Lq, 02.60.Pn
\end{abstract}

Magnets and superconductors are examples of physical systems where quenched disorder often plays a dominant role. Such systems can exhibit hysteresis effects and long relaxation times that are the manifestation of the large energy barriers created by the quenched disorder. One scenario that makes predictions for the equilibrium and nonequilibrium behavior of disorder dominated phases is the droplet or scaling picture [1]. Predictions in this scenario follow from scaling assumptions for the energetic and geometric properties of excitations. For simple topological reasons, excitations can be defined as regions where the configuration is uniformly related by a symmetry to a global ground state (e.g., spin flipped domains.) In the droplet picture, the low lying excitations of size $l$ are connected and compact: they have volume $\sim l^{d_{f}}$, with dimension $d_{f}$ equal to the system dimension $d$, and the surface to volume ratio decreases as $l$ increases. Droplet boundaries are fractal, with surface dimension $d_{s}<d$. The central ansatz is that the probability distribution $\rho(\Delta, l)$ for the energy $\Delta$ of a droplet of size $l$ in a given volume $\sim l^{d}$ has a characteristic scale $\sim l^{\theta}$. This distribution is argued to have finite weight at $\Delta=0$. The two exponents $\theta$ and $d_{s}$ can be used, for example, to predict many of the properties of a spin glass [1]. This scenario is consistent with numeric results for excitations created by modifying boundary conditions [2, 3]. However, other work 沺 6] has suggested that there may be more than one important energy scaling exponent and more complicated geometries for excitations. The proposed distinct exponents separately describe (i) boundary induced domain wall excitations and (ii) excitations induced by internal constraints or external fields. It has also been suggested that there is distinct scaling for large droplets created by modifying the quenched disorder [7]. It is important to understand these claims, as they suggest that the standard droplet picture is, at best, incomplete.

To provide perspective, it is useful to investigate in detail systems which lend themselves to precise study, where some analytic results are known and large systems can be simulated efficiently. Results are presented here for a 2D elastic medium and a 2D Ising spin glass. Single interior droplets, which include a specified central point, are computed for the elastic medium. In contrast with work on interior droplets in 2D spin glasses [4], a fast, exact algorithm is used, allowing for precise checks of scaling. The responses of the elastic medium and the spin glass to bulk perturbations are also calculated. The numerical results for droplet energies and geometrical characteristics show that logarithmic or small power law corrections are quite strong. These corrections can be understood in detail by arguments within the droplet picture. Droplets that are not induced by boundary conditions are only bounded above by the system size $L$ and below by a discretization scale, so that all scales between must be considered when computing averages. Corrections to scaling for droplets of fixed scale $l$, such as $l^{-1}$ or $L^{-1}$ corrections (e.g., from lattice discreteness) or unknown irrelevant operators, might also be considered. However, the scale averaging corrections are apparently dominant for some quantities. Such corrections lead to an effective energy exponent distinct from $\theta$, as boundary condition induced domain walls do not have such corrections. To remove scale averaging corrections, one can group the droplets by scale $l$ and study the geometry and energy as a function of $l$ (or $l / L$ if one is interested in large droplets [6]), as $L \rightarrow \infty$. With this analysis, the numerical results provide strong evidence that the droplets are "compact", with fractal domain walls, and that there is a single energy exponent $\theta$.

One model that I study here is for a two dimensional elastic medium, with scalar displacement field $u(x)$, interacting with quenched periodic disorder. The continuum energy functional is $\mathcal{H}[u(x)]=\int d^{2} x[\nabla u(x)]^{2}+$ $V(u(x), x)$, where $V$ has short range correlations in its second argument and is periodic in its first argument, $V(u(x)+1, x)=V(u(x), x)$. This model has been used for vortex lattices in superconductors, incommensurate charge density waves, and crystal growth on a disordered substrate [8]. The continuum model can be discretized 
on a scale $a$, where the disorder and elastic energies balance [9]. As an effective degree of freeedom $i$ is pinned to a preferred configuration (up to periodic shifts), the displacements $u_{i}$ are of the form $n_{i}+\beta_{i}$, for integer $n_{i}$ and fixed $\left\{\beta_{i}\right\}$. Elastic interactions tend to minimize nearest neighbor differences in $u_{i}$, with excitations of the medium being regions displaced relative to the ground state. Since the $u_{i}$ are discretized, domain walls separate regions relatively shifted by unit amounts. Numerical work for zero temperature $(T=0)$ has determined properties of the ground state and the scaling of boundary induced domain wall energies [10,11, 13, 12]. Finite temperature simulations [14], both Monte Carlo and combinatorial, have shown that the $T=0$ phase is stable at finite $T$. This model is thus a useful prototype for models with finite $T$ transitions, such as the $3 \mathrm{D}$ spin glass 15 .

Another model treated here is the 2D Ising spin glass, with Hamiltonian $\mathcal{H}=-\sum_{\langle i j\rangle} J_{i j} s_{i} s_{j}$, with spins $s_{i}=$ \pm 1 on a triangular lattice and Gaussian distributed $J_{i j}$. The ground states $\left\{s_{i}^{0}\right\}$ for samples in this model were found by a combinatorial method for a standard graph representation [11,16].

For the elastic medium, minimal energy domain walls about the center of a sample were studied on a square lattice using a polynomial time algorithm [17 that calculated the energy $\Delta_{o}$ and the droplet boundary. One method to characterize the compactness of droplets is to compare $R_{O}$, the radius of the smallest circle that encloses the droplet, with $R_{I}$, the radius of the largest circle contained by the boundary vertices. Droplets can be studied in spin glasses by finding the ground state and then recomputing the ground state $\left\{s_{i}^{\epsilon}\right\}$ with modified couplings $J_{i j} \rightarrow J_{i j}-\epsilon L^{-d} s_{i}^{0} s_{j}^{0}$ [18], 7]. This bulk perturbation can introduce excitations on all scales. Sample excitations are depicted in Fig. 11.

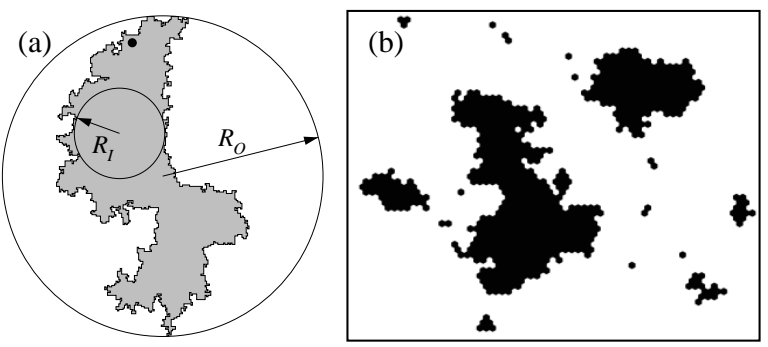

FIG. 1. (a) Diagram of the geometry of a sample droplet in the $2 \mathrm{D}$ elastic medium. The droplet is the region which can be displaced with minimal energy cost and contains the sample center (dot). The radii $R_{O}$ and $R_{I}$ are defined in the text. (b) Droplets induced by a bulk perturbation $(\epsilon=16)$ in a $64^{2}$ spin glass sample. The filled areas have spins flipped relative to the unperturbed ground state $\left(s_{i}^{o} \neq s_{i}^{\epsilon}\right)$. For the results shown in Fig. A, $\epsilon \approx 0.35,1.3$ and droplets rarely intersect.

The droplet energies are of great interest, as these are believed to determine the static correlation functions at finite temperature and the relaxation to equi- librium. Consider the problem of finding the minimal energy droplet around the origin in a system of size $L$ [4]. Assume that there is a factor $b$ which gives a separation of length scales: droplets differing in size by $b$ are independent. One independent droplet excitation could be excited at each scale, so that sums over all scales must be performed (for similar sums, see Refs. 19,18,6].) At each length scale $s, s=1,2, \ldots, \log _{b}(L)$, the distribution for the energy $\Delta\left(b^{s}\right)$ has characteristic scale $b^{s \theta}$ and has finite weight $b^{-s \theta}$ at $\Delta=0$ [1]. The total density of states $\rho\left(\Delta_{o}\right)$ for $\Delta_{o}<L^{\theta}$ is then a sum over $s$, giving $\rho\left(\Delta_{o}\right) \sim L^{-\theta}\left|1-\left(b^{\prime} L\right)^{\theta}\right|$, for $\theta \neq 0$, where $b^{\prime}>0$ is set by $b$ and the lattice and boundary conditions, which affect the lower and upper ends of the sum. The expected minimum value for $\Delta_{o}$ scales as $\sim L^{\theta}\left|1-\left(b^{\prime} L\right)^{\theta}\right|^{-1}$, the subdominant term reflecting that the minimal energy droplet is chosen from all length scales from 1 to $L$. The effective energy exponent is then (for $\theta<0$ )

$$
\theta^{\mathrm{eff}}=\frac{d \ln \left(\overline{\Delta_{o}}\right)}{d \ln (L)}=\frac{\theta}{1-\left(b^{\prime} L\right)^{\theta}} .
$$

Applying Eqn. (1) to the 2D spin glass, taking $b^{\prime}=2$ and $L=16$, gives an effective exponent $\theta^{\text {eff }}=-0.45$, apparently quite different from the domain wall value $\theta=-0.28$ 2] and consistent with the alternate energy exponent proposed in earlier numerical work [4]. The effective exponent converges to $\theta$ quite slowly with $L$ (and is relatively insensitive to $b^{\prime}$ ), as $\theta$ is near zero.

One case where $\theta=0$ for domain walls created by boundary conditions is the $2 \mathrm{D}$ elastic medium. Large domain walls can be created by external strains. By statistical tilt invariance of the disorder 20], the change in the sample averaged energy can be found by computing the elastic energy only, as the change in the sample averaged pinning energy is zero. Displacing one end of a sample by $\delta u=1$ to induce one domain wall gives an elastic energy density $\sim L^{-2}$ over the volume $L^{2}$, so that the total domain wall energy scales as a constant $(\theta=0$.) This result is consistent with previous numerical simulations of boundary induced domain walls [10]. However, the mean interior droplet energy $\overline{\Delta_{o}}(L)$ can be fit over a decade with $-0.15<\theta<-0.23$, to within a few percent for smaller $L$. Arguments similar to those for $\theta \neq 0$ can be applied to explain this. There are $\ln (L / a) / \ln (b)$ independent scales to choose from, each with identical droplet energy distributions $(\theta=0)$. The inverse of the minimal droplet energy $\left[\overline{\Delta_{o}}(L)\right]^{-1}$ is therefore linear in $\ln (L)$. This result can also be derived using elasticity theory. The displacement at the origin of a region of size $a$ costs an elastic energy that scales as $\sim[\ln (L / a)]^{-1}$. By tilt symmetry, the pinning can be averaged over, so that $\overline{\Delta_{o}}(L) \propto[\ln (L / a)]^{-1}$ for interior droplets constrained to contain the origin. The numerical results are quite consistent with these expectations, as shown by the two parameter fit displayed in Fig. 2 (in addition, the computed 
probability of generating a droplet of size $l$ is consistent with a distribution uniform in $\ln (l)$ [17.)

Similar corrections are important for the magnetization $m(h)$ of a spin glass in response to an external field $h$ [1]. For the case $\theta \neq 0$ and $h<O\left(L^{\theta-d / 2}\right), m$ is found by summing over scales the product of the probability $h l^{d / 2-\theta}$ of generating a droplet, its expected contribution $l^{d / 2} L^{-d}$ to the magnetization, and the number of droplets $(L / l)^{d}$ at scale $l$. This gives $m \propto h\left|\left(b_{h}^{\prime} L\right)^{-\theta}-1\right|$, with $b_{h}^{\prime}>0$ a constant, to be compared with the uncorrected singular piece $m \propto h L^{-\theta}$ (for numerics, see Ref. [5]). The size of the corrections are quite similar to those for $\theta$.

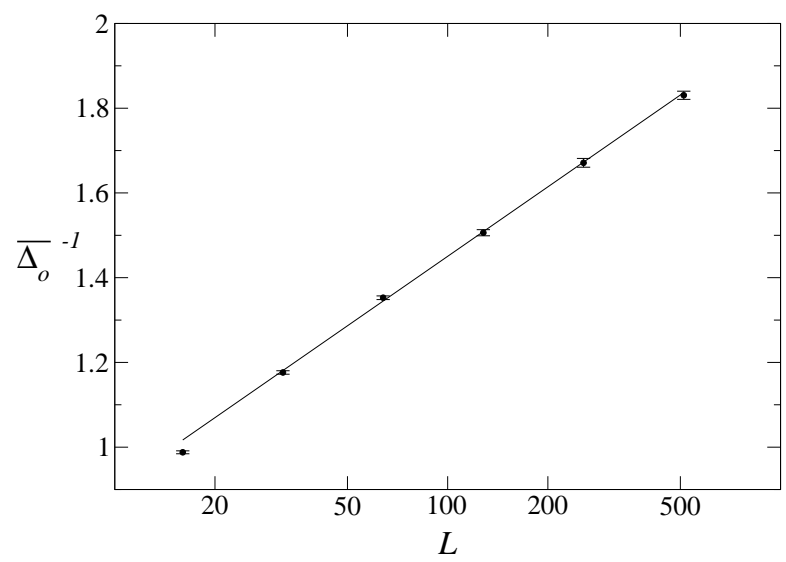

FIG. 2. Plot of the inverse of the mean droplet energy $\overline{\Delta_{o}}$ vs. system size $L$ in the $2 \mathrm{D}$ elastic medium, averaged over at least $10^{4}$ samples for each $L .1 \sigma$ error bars are shown. The line indicates the fit $\Delta_{o}(L)=[0.363+0.263 \ln (L)]^{-1}$ for $512 \geq L \geq 32$, with $\chi^{2}=1.3$ for 3 d.o.f.

The measurement of geometrical quantities, such as boundary length and droplet area, can also be strongly influenced by scale averaging corrections if one averages over all length scales from 1 to $L$. When the sample averaged area $\overline{A(L)}$ of interior droplets in the 2D elastic medium is computed as a function of $L$ and the local exponent $d_{\bar{f}}^{\mathrm{eff}}=d[\ln (\overline{A(L)})] / d[\ln (L)]$ is computed, the local dimension is less than two, which might suggest fractal droplets. This local exponent slowly changes with $L$, though (Fig. 3.) The local exponent for $\overline{A(L)}$ is $d_{\bar{f}}^{\text {eff }}=2-\left[\ln \left(L / a^{\prime}\right)\right]^{-1}+O\left(L^{-2}\right)$, where $a^{\prime}$ depends on the boundary and lattice cutoffs. A useful procedure to reduce the corrections to $d_{f}^{\text {eff }}$ is to separate out the scales and plot the droplet area $A\left(R_{O}, L\right)$ as a (binned) function of $R_{0}$. Changing the order of the averages gives local exponents that are much better fit by a constant. For $L>32$ and $R_{0}>8, \overline{A\left(R_{o}\right)} \sim R_{o}^{d_{f}}$, with bulk droplet dimension $d_{f}=2.01(2)$ (Fig. [3].) A similar plot for the perimeter confirms [11] that $d_{s}=1.25(1)$, with the surface to volume ratio vanishing as $l^{d-d_{s}}$ for large droplets. Droplet compactness can also be confirmed by plotting the ratio $k=R_{O} / R_{I}$ binned according to $R_{O}$; it is found that the distribution of $k$ converges, with mean $\left\langle k\left(R_{O}, L\right)\right\rangle=2.85(5)$, when $L / 2>R_{O}>32$.

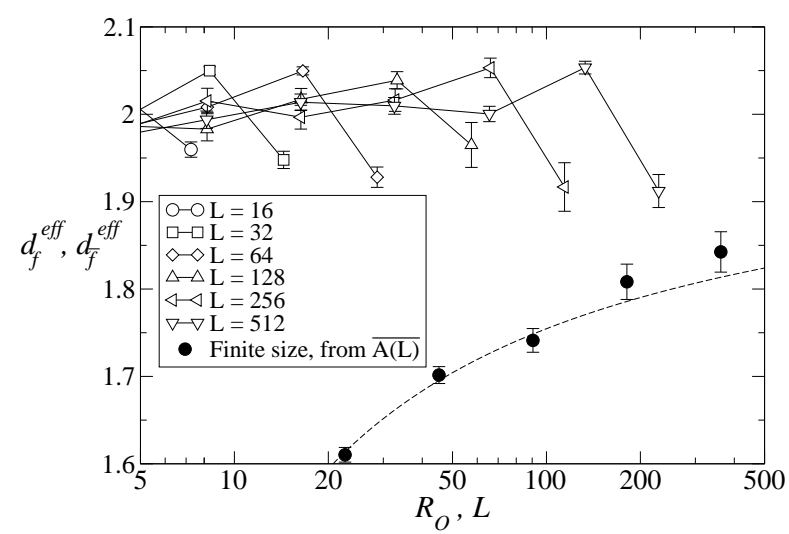

FIG. 3. Estimates of the droplet fractal dimension in a 2D elastic medium ( $1 \sigma$ statistical uncertainties shown.) Fixing $L$, binning droplets by linear size $R_{O}$, and computing the discrete logarithmic derivative to estimate $d[\ln (A))] / d\left[\ln \left(R_{O}\right)\right]$ gives the droplet dimension $d_{f}^{\text {eff }}\left(R_{O}\right)$ values connected by the solid lines. From this method $d_{f}=2.01(2)$. Averaging droplet area $A$ over samples and computing the discrete logarithmic derivative to estimate $d[\ln (\bar{A})] / d[\ln (L)]$ gives the finite size estimate $d \frac{\mathrm{eff}}{f}(L)$. The dashed line shows a fit using the correction derived in the text with $a^{\prime}=1.7(3)$.

Corrections to geometric and energetic quantities are also important when computing link overlaps, such as those found in comparing the unperturbed $J_{i j}$ ground state, $\left\{s_{i}^{0}\right\}$, with the $\epsilon$-perturbed state. The link overlap $q_{l}$ is the fraction of link values $s_{i} s_{j}$ on bonds $\langle i j\rangle$ which are unchanged. By summing the contributions over all scales (note the small droplets in Fig. 11(a)), it can be shown that the local exponent for the fraction $1-q_{l}$ of changed bonds behaves as

$$
\mu_{l}^{\mathrm{eff}}=-\frac{d\left[\ln \overline{\left(1-q_{l}\right)}\right]}{d \ln (L)}=\mu_{l}-\frac{c^{\prime}}{(c L)^{d-\mu_{l}}-1},
$$

where $\mu_{l}=\theta+2\left(d-d_{s}\right)$ and $c, c^{\prime}$ are constants characterizing the upper and lower cutoffs. The computed local exponent for the $2 \mathrm{D}$ spin glass is shown in Fig. $14\left(\mu_{l}^{\mathrm{eff}}\right.$ is relatively insensitive to $\epsilon$, at least for $0.35<\epsilon<1.3$.) Only for $L>100$ does $\mu_{l}^{\text {eff }}$ approach the large $L$ limit of $\mu_{l} \approx 1.18$ (using the values $\theta=-0.28$ and $d_{s}=1.27$.) Similar results are found for the $2 \mathrm{D}$ elastic medium [17]. The exponent $\mu$ for spin overlaps $q=L^{-d} \sum s_{i}^{o} s_{i}^{\epsilon}$, with $1-q \sim L^{-\mu}$, has smaller corrections of this form and may well be dominated by corrections to scaling from unknown operators or inverse lengths. Numerics 17 show that $\mu$ converges much more quickly than $\mu_{l}$ in the $2 \mathrm{D}$ spin glass. The measurement of $\mu_{l}$ (relative to $\mu$ ) has been used by Palassini and Young [7] to conclude that a second energy exponent $\theta^{\prime}$ affects the response to bulk perturbations in the $3 \mathrm{D}$ spin glass. In three dimensions, the scale averaging corrections decrease more 
quickly with $L$, as $d-\mu_{l} \approx 1.3$ compared with the $2 \mathrm{D}$ correction exponent $d-\mu_{l} \approx 0.82$, but the system sizes that can be simulated are much smaller. It may be that corrections due to irrelevant variables or possible $1 / L$ effects are dominant, but scale averaging corrections clearly contribute to errors in $\mu_{l}$. A correction of $\delta \mu_{l} \approx-0.2$ for $L \approx 8$, from similar $c$ and $c^{\prime}$, would invalidate the conclusions of Ref. |7].

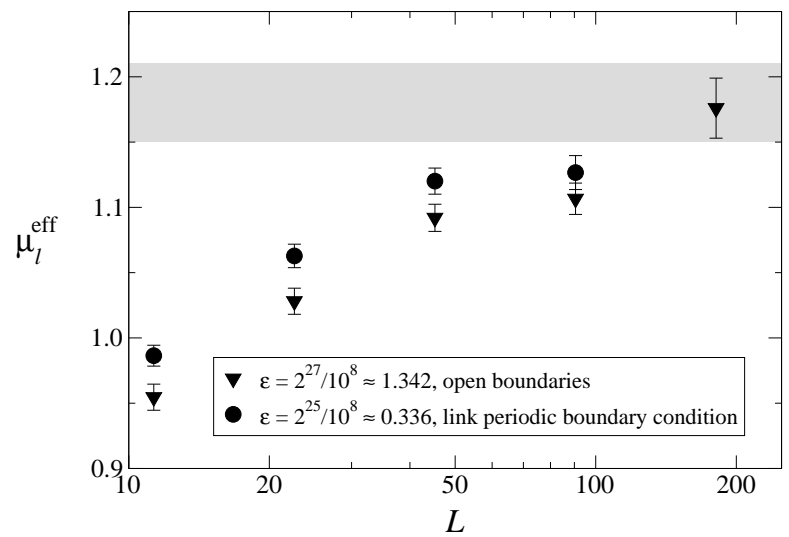

FIG. 4. Plot of the local exponent $\mu_{l}^{\text {eff }}$ (from the discrete derivative between $L=8,16, \ldots, 256)$ for the link overlap $q_{l}$ in the $2 \mathrm{D}$ spin glass as a function of system size. Results are shown for distinct $\epsilon$ and boundary conditions (open, with boundary spins fixed under perturbation, and link periodic.) The expected limit at large $L$ is $\mu_{l}=1.18(3)$ (shaded region.)

In summary, analysis of numerical data provides a precise confirmation of the droplet picture in the bulk of a sample, both for the scaling of the energies and for the geometrical structure of droplets. In comparing the numerical results with the droplet picture, care must be taken to understand where strong corrections might arise. The corrections arising from averaging over multiple scales can be predicted in some detail and apply to spin glasses and other models where $\theta$ is near zero. The corrections to averaged quantities such as the droplet energy $\overline{\Delta_{o}}(L)$ are satisfactorily explained, for $L^{2}>10^{2}$ systems, by averaging simple power laws over scales between 1 and $L$. The link overlap, which inherently averages over scales, is strongly affected by finite size corrections for small $\theta$ and $d-d_{s}$, with effective exponent corrections greater than 0.1 for $L<30$ in the $2 \mathrm{D}$ spin glass. It has been suggested that, for topological reasons, distinct $\theta$ exponents exist only in $d \geq 3$ [6]. The numerics in this Letter are for $d=2$, but they and the general analysis suggest that large finite size effects strongly affect results in three dimensions. To reduce these types of corrections, data can be binned over droplet size $l$ (or over $l / L$ ) at fixed system size $L$, checking for convergence by then increasing $L$.
I would like to thank David Huse for bringing to my attention some recent work on droplets, Olivier Martin for a stimulating discussion, and Daniel Fisher for discussions. The LEDA library 21] was most useful in the study of the geometry of the droplets. This work was supported by the National Science Foundation (DMR9702242).

[1] D. S. Fisher and D. A. Huse, Phys. Rev. Lett. 56, 1601 (1986); Phy. Rev. B 38, 386 (1988); A. J. Bray and M. A. Moore, in Heidelberg Colloquium on Glassy Dynamics and Optimization, L. Van Hemmen and I. Morgenstern eds. (Springer-Verlag, 1986); W. L. McMillan, J. Phys. C 17, 3179 (1984).

[2] A. J. Bray and M. A. Moore, J. Phys. C 17, L463 (1984); W. L. McMillan, Phys. Rev. B 29, 4026 (1984); H. Rieger, et al, J. Phys. A 30, 3939 (1997).

[3] D. McNamara, A. A. Middleton, C. Zeng, Phys. Rev. B 60, 10062 (1999).

[4] N. Kawashima and M. Suzuki, J. Phys. A 25, 4985 (1992); N. Kawashima and T. Aoki, cond-mat/9911120.

[5] H. Rieger, et al, J. Phys. A 29, 3939 (1996).

[6] J. Houdayer and O. C. Martin, Phys. Rev. Lett. 81, 2554, 1998; Euro. Phys. Lett. 49, 794, 2000. F. Krzakala and O.C. Martin, cond-mat/0002055.

[7] M. Palassini and A. P. Young, cond-mat/0002134.

[8] J. Toner and D. P. DiVincenzo, Phys. Rev. B 41, 632 (1990); T. Hwa and D. S. Fisher, Phys. Rev. Lett. 72, 2466 (1994).

[9] Y. Imry and S.-K. Ma, Phys. Rev. Lett 35, 1399 (1975).

[10] C. Zeng, A. A. Middleton, and Y. Shapir, Phys. Rev. Lett. 77, 3204 (1996); A. A. Middleton, Phys. Rev. E52, 3337 (1995).

[11] A. A. Middleton, Phys. Rev. B 61, 14787 (2000).

[12] C. Zeng, P. L. Leath, and D. S. Fisher, Phys. Rev. Lett. 82, 1935 (1999).

[13] H. Rieger and U. Blasum, Phys. Rev. B 55, R7394 (1997).

[14] G. G. Batrouni and T. Hwa, Phys. Rev. Lett. 72, 4133 (1994); C. Zeng, P. L. Leath, and T. Hwa, Phys. Rev. Lett. 83, 4860 (1999).

[15] K. Binder and A. P. Young, Rev. Mod. Phys. 58, 801 (1986); Spin Glasses and Random Fields, A. P. Young ed. (World Scientific, Singapore, 1998).

[16] F. Barahona, J. Phys. A 15, 3241 (1982).

[17] A. Alan Middleton, in preparation.

[18] B. Drossel, H. Bokil, A. J. Bray, and M. Moore, Eur. Phys. J. B 13, 369 (2000).

[19] D. S. Fisher, Phys. Rev. Lett. 78, 1964 (1997).

[20] U. Schulz, et al, J. Stat. Phys. 51, 1 (1988).

[21] http://www.mpi-sb.mpg.de/LEDA. 